\section{Aporte del coaching en la toma de decisiones gerenciales}

decision making
Andrés Felipe Jiménez-López ${ }^{1,2}$

andresfjl@gmail.com

https://orcid.org/0000-0002-9361-1112

María Paulina Vásquez-Varela ${ }^{1}$

mapalinav@gmail.com

https://orcid.org/0000-0002-7577-9149

Doralba Ramírez Henao ${ }^{3}$

doralba30@gmail.com

https://orcid.org/0000-0003-1802-5233

https://doi.org/10.22209/rhs.v8n1a05

Recibido: marzo 23 de 2020.

Aceptado: junio 17 de 2020.

\section{R e s u m e n}

El presente artículo tiene como propósito establecer el aporte del coaching en la toma de decisiones, una de las habilidades gerenciales de mayor demanda en la gestión administrativa. Se ponen en diálogo dos perspectivas, una desde el coaching organizacional, proceso conducente a generar cambios positivos y duraderos para agenciar la ruta del éxito en los equipos de trabajo y, la segunda, desde las decisiones gerenciales, mediante el desarrollo consciente de habilidades y recursos que permitan conectar las emociones con la razón, en la búsqueda de alternativas para elegir la mejor

1 Corporación Universitaria Remington.

2 Corporación Universitaria de Santa Rosa de Cabal - Unisarc. 3 Independiente. opción a emprender. Esto sugiere la cualificación desde el ser, saber y hacer, pues si bien, los conocimientos técnicos y competencias relacionadas con el desempeño de un cargo son necesarios, es indispensable gestionar nuevos aprendizajes personales y profesionales, donde el coaching se configura como una opción válida para facilitar dicho camino. Por tanto, se destaca que el coaching aporta de forma efectiva, mediante un proceso profundo de transformación personal, en la toma de decisiones, puesto que al alcanzar mayores niveles de consciencia y de conexión interna, se amplifica la capacidad de evaluar integralmente las situaciones, con mayor asertividad al momento de elegir, lo cual se traduce en resultados óptimos.

Palabras clave: coaching; gerencia; habilidades gerenciales; toma de decisiones.

\section{Abstract}

The purpose of this paper is to determine the extent of the contribution of coaching in decision making, one of the most sought-after managerial skills in administrative management. Two perspectives are brought into dialogue in this paper. One from organizational coaching, a process leading to positive and lasting changes to pave the way for success in work teams. And the second one from managerial decisions, through the conscious development of skills and resources that allow linking emotions and reason, in the search for 
alternatives to choose the best option to undertake. This entails qualification of different aspects of the being, the knowing and the doing. While technical knowledge and skills associated to a position are necessary, it is essential to undertake new personal and professional experiences. Here, coaching is a valid option for paving the way for success. Therefore, it is highlighted that coaching effectively contributes, through a deep process of personal transformation, to decision-making, because when higher levels of awareness and internal connection are reached, the ability to fully evaluate situations is expanded, and more assertiveness to choose is achieved, resulting in superior outcomes.

Keywords: coaching; management; managerial skills; decision making.

\section{Introducción}

La gestión integral de las organizaciones exige el desarrollo de competencias claves en la alta gerencia, donde la toma de decisiones efectivas constituye un elemento de valor para enfrentar los desafíos constantes que plantea un mundo globalizado y competitivo. Lo anterior, sugiere un proceso secuencial y cualificado de empoderamiento, que genere virtuosidad desde el aprendizaje individual y capacidad de integración en el contexto organizacional, donde es vital el camino de autodescubrimiento y movilización de esquemas internos, dirigido desde el coaching para la generación de procesos transformacionales.

Para el abordaje reflexivo se orienta la discusión a partir de cuatro componentes dentro de la estructura temática: el primero es el coaching y la gerencia, como una metodología innovadora y e ficaz para el desarrollo personal y profesional. El segundo componente, se desarrolla alrededor de las tendencias en el coaching organizacional destacando que, cada vez, toma mayor fuerza la gestión de equipos altamente efectivos y esto requiere herramientas de alto impacto para movilizar capacidades. El tercer capítulo aborda las habilidades gerenciales, desde el enfoque de un desempeño exitoso para liderar personas que acompañan el logro de los objetivos organizacionales.

Por último, se trata la toma de decisiones, como una habilidad fundamental en cualquier actividad humana, pero con una amplia repercusión cuando se tiene esta exigente tarea a nivel empresarial; motivo por el cual es necesario disponer de herramientas que guíen la gestión efectiva y consciente de dicho proceso, donde el coaching surge como una metodología inspiradora en la forma de enfrentar, con poder creativo, la toma de decisiones y estar preparado para asumir diferentes retos con un óptimo desempeño.

\section{El coaching y la gerencia}

"El coaching ha experimentado un gran desarrollo como disciplina para promover el cambio y el bienestar en personas y organizaciones de todo el mundo» (González et al., 2018, p. 81). Así se destaca la fuerza que ha venido tomando este proceso expansivo de las capacidades individuales y colectivas, donde las personas logran situarse como autogestionarias de su vida, de sus experiencias, amplían la valoración del potencial que pueden llegar a desarrollar, con repercusión en los entornos cotidianos y en la forma de gestionar las relaciones, sustento vital de lo humano y de lo social. 
Ahora bien, al hablar de coaching y gerencia, se plantean retos, algunos de los cuales están directamente asociados a las nuevas demandas directivas, como señala Echeverría (2000) citado en Leal et al. (2017): «El directivo de la empresa del futuro requerirá disponer de las competencias de un coach [...] debe ser alguien capaz de identificar los obstáculos que obstruyen el desempeño y aprendizaje de su gente y desarrollar intervenciones capaces de disolverlos» (p. 131). De esta manera, resulta comprensible que, para hablar de la tarea gerencial, se requiera un compromiso con el bienestar propio y el de las personas que acompañan el logro de los resultados organizacionales, destacando la importancia de encontrar la ruta para ejecutar un trabajo colaborativo e interdependiente.

Así, toma validez la exploración de un entorno estratégico que cualifique el desempeño gerencial, más allá de los procesos administrativos y funcionales, donde se puede apreciar un campo de intervención bien definido para el coaching, como una práctica generadora de cambios, que incrementa las opciones de aprendizaje y transformación de cada persona y de los equipos. «El coaching está dirigido a motivar o influenciar en el grupo que hace las cosas para convertirlo en un equipo de trabajo donde todos se lleven de forma armoniosa y el trabajo sea el más productivo posible» (Recuenco, 2018, p. 83). Lo anterior, refuerza la relación sinérgica que existe entre el arte de gerenciar y la habilidad de liderar, así como afırma Navia: «Los gerentes más eficaces se convierten en líderes y para llegar a ello, se han identificado dos factores críticos para el éxito; el aprendizaje y la participación del directivo» (Navia, et al., 2019, p. 1077).
Innegablemente, esto requiere un aprendizaje constante y continuo, que implica la participación del individuo, más allá de un simple espectador, con verdadera injerencia en las decisiones que se toman, la direccionalidad que se busca dar al proyecto de vida y su acoplamiento con la gestión gerencial, que vincula el esfuerzo de las personas para cumplir las metas y objetivos trazados.

Como afirman Sánchez y Boronat: «A lo largo de su trayectoria, el coaching se ha convertido en una herramienta clave y exitosa en el management empresarial y su influencia se ha extendido a diversos ámbitos» (Sánchez y Boronat, 2014, p. 223). Esto denota una transición importante hacia nuevos modelos gerenciales, desde la sinergia de talentos y la ampliación de los escenarios para el encuentro colectivo, con líderes que logren asumirse desde su rol fundamental como agentes de cambio, facilitadores del aprendizajes y gestores de oportunidades que se traduzcan en bienestar para las personas.

Un líder coach es un guía del proceso, busca enseñar y corregir frente a un problema, trabaja en equipo fomentando la toma de decisiones y el entusiasmo, visualizando a las barreras como oportunidades de mejora, teniendo autoridad como un privilegio del servicio. (Suárez, 2020, p. 240)

En efecto, se habla de gerentes que logren trascender la verticalidad que suele representar su rol en la organización, hacia un modelo de gestión más centrado en relaciones de complementariedad e interdependencia, con aprecio hacia la visión transformadora que se necesita para llevar a niveles superiores los emprendimientos empresariales. 
El coaching busca desarrollar ese potencial que tiene cada persona y que desconoce, para utilizarlo en su beneficio personal y grupal, en donde las capacidades para dar soluciones son más obvias y fáciles de implementar, ya que esta metodología de cierta manera hace que el individuo despierte y observe mejor cada paso en el proceso de entendimiento humano. (Díaz, 2016, p. 122)

Gerencia y coaching, un vínculo generativo, un camino de complementariedad en la búsqueda necesaria de opciones de bienestar, donde quienes están al frente de la labor directiva tienen una función primaria de profundizar en los talentos de las personas y acompañarlas en los procesos para que puedan lograr sus metas y obtener resultados extraordinarios. Esta articulación representa una alternativa de valor para mejorar el desempeño organizacional, a partir de un proceso de aprendizaje colectivo y el alineamiento en torno a una visión compartida.

\section{Tendencias en coaching organizacional}

En los últimos años la sociedad ha experimentado cambios importantes y acelerados, con un alto impacto en las relaciones y en la gestión gerencial, lo cual, a su vez, implica incorporar modelos de desarrollo organizacional más centrados en las personas, así como sistemas directivos enfocados en el bienestar y la complementariedad de los equipos de trabajo. En este contexto, el coaching organizacional se configura como una tendencia global, que ha logrado un alto reconocimiento como una metodología de entrenamiento personal y empresarial.

Al respecto Goldvarg y Perel precisan que, «en Latinoamérica, los individuos y las organizaciones están aprovechando el coaching para mejorar su rendimiento laboral, ampliar sus oportunidades profesionales y mejorar estrategias de gestión empresarial» (citado en $\mathrm{Ca}$ sique, 2018, p. 15). En esa misma dirección, «desde la década de los 90 hasta la fecha, el coaching se ha establecido como una nueva profesión, que a nivel organizacional apoya procesos de gestión, fortalece los resultados y el trabajo en equipo, así como también acompaña a mejorar la calidad de vida de las personas» (Castillo y Medina, 2016, p. 69).

De este modo, el coaching aparece como un elemento vocacional en la administración actual, toda vez que acompaña cambios en los grupos humanos y da lugar a nuevas formas de dirigir y repensar la función gerencial, y dentro de esta, el conjunto de relaciones que se moldean en la conducción de los equipos de trabajo.

Sin embargo, existen otras tendencias que tienen una buena presencia a nivel de la gestión de equipos, como mindfulness, gamificación, neuroliderazgo, entre otros, cuyo campo de intervención aporta al mundo profesional, a la identificación de la contribución de las personas como parte de un equipo, a la generación de entornos de bienestar y la promoción de una cultura de innovación, colaboración y mejora continua. "Si bien, las técnicas mencionadas, son básicamente procesos de apoyo, estos pueden complementar ampliamente otras mega-tendencias administrativas, especialmente 
aquellas enfocadas al desarrollo personal de los integrantes de la organización» (Aponte, 2017, p. 20).

En general, las acciones encaminadas al mejoramiento continuo de los procesos son determinantes para la competitividad y la productividad; sin embargo, lo administrativo se vale de lo humano, se gestiona desde los equipos de trabajo, de allí la necesidad de fortalecer la cultura organizacional. «El mundo empresarial en la actualidad está acomodando su cultura organizacional y se está orientando hacia el bienestar de sus colaboradores y al desarrollo de su proyecto de vida basado en un equilibrio correcto entre vida laboral y vida personal» (Castillo y Medina, 2016, p. 73).

Por lo expuesto, es importante dimensionar lo humano a partir de un enfoque de desarrollo integral, donde el espacio laboral sea una fuente revitalizadora para quienes se encuentran en este. Así, retomando lo que dice Mu$\operatorname{sicco}(2015)$ :

En las organizaciones es útil para acelerar el desarrollo de las competencias de cada directivo y de los grupos, estimular motivación, mejorar el clima de la empresa y las prestaciones para conseguir nuevos objetivos en las fases de cambio de la organización. (p. 127)

Desde este referente y sin desconocer los procesos de cambio que deben liderarse, la gestión directiva tiene un reto importante y es que cada persona se sitúe en el hacer, desde el ser, con el propósito de hallar un sentido superior que asegure una alta movilidad hacia lo organizacional.
Surge desde este pensamiento la conexión con los sentidos de vida, «ver la importancia que cobra para un gerente el proceso interactivo, en donde se va a conocer a sí mismo, conocer su espacio emocional, sus potencialidades, sus limitaciones y aprender a gerenciar y desde allí, puede aportar más a los demás» (Romero, 2012, citado en Díaz, 2016, p. 116).

Básicamente se trata de romper estructuras, paradigmas, pasar de lo netamente funcional, hacia la complejidad que reviste sintonizar los lenguajes humanos y establecer vínculos generativos para la consecución de las metas organizacionales.

Para emprender el camino hacia el éxito empresarial, se requiere visión y gran un talento para inspirar a los otros, «desarrollar a las personas enfocándose en su crecimiento laboral y personal, empedrando a los mismos por medio de libertad en la toma de decisiones» (Suárez 2020, p. 240). Así, al movilizar la capacidad de expresión de los recursos individuales se tiene como resultado, casi inequívoco, una inteligencia amplificada, desde lo emocional y racional, incrementando a su vez la capacidad de evaluar de forma integral los procesos conducentes a la toma de decisiones, en donde la libertad, vista en un sentido de autonomía y autodeterminación, aseguran una mayor efectividad.

\section{Como bien menciona Arroyo (2013):}

Es importante aceptar, que el éxito de una organización está en el éxito de su gente. Hay que reconocer también que esta responsabilidad no puede recaer en unos cuantos gerentes. La innovación tiene que ser permanente y general esto solo se logra con el liderazgo colectivo. (p. 3) 
En tal sentido y como lo plantean Chew et al., (2020): «El apoyo y el compromiso de la alta dirección en el desarrollo de una cultura corporativa que hace hincapié en la apertura, el aprendizaje y el desarrollo son muy críticos para el éxito del coaching» (p. 12). Desde esta mirada, es perceptible una mayor apertura para integrar dentro de la gestión gerencial y de los equipos colaborativos, nuevas metodologías para el desarrollo de las habilidades blandas, contexto donde aparece el coaching. No obstante, existen también algunas posturas que señalan vacíos a nivel metodológico y procedimental, que pueden repercutir en la rigurosidad del proceso transformacional esperado. Al respecto, Baquero y Rodríguez (2016) plantean:

El coaching ha tenido un importante crecimiento en los últimos años como método que facilita el logro de metas y propicia el desarrollo de habilidades. Sin embargo, existen críticas dirigidas, por un lado, a la débil fundamentación teórica que sustenta su proceso y, por otro, a la baja producción de investigaciones empíricas que expliquen los resultados de éxito y que muestren con rigor los cambios cognitivos, motivacionales y afectivos logrados por las personas que participan en este tipo de procesos (p. 141).

En esta misma línea, Grant (2011) «reconoce la importancia de desarrollar estrategias y acciones que permitan la evaluación del proceso de coaching, con el fin de satisfacer dos necesidades actuales: por un lado, demostrar su eficacia como método y, por otro, explorar el proceso, examinando cuáles son las variables intervinientes» (citado por Baquero \& Rodríguez, 2016, p. 144).
Aunque son destacadas las ventajas y beneficios que ofrece esta disciplina, es importante acoplar y consolidar algunos elementos del modelo, sin desconocer que existe un método que empodera a las personas y organizaciones en los procesos de cambio, sí debe lograrse un ejercicio evaluativo con validez técnica.

Como elementos finales, se destaca el coaching como una tendencia en auge, más no unitaria, pues están en medio otras metodologías, disciplinas y corrientes que resultan complementarias y efectivas a la hora de abordar la complejidad del ser humano. Cuenta con un reconocido valor para empoderar a las personas y los equipos de trabajo desde la corresponsabilidad, que acompañada de los nuevos modelos gerenciales logra escalar hacia el desarrollo de las capacidades individuales hacia un liderazgo positivo, inspirador e integrador del potencial colectivo.

\section{Habilidades gerenciales}

Un desempeño exitoso es la meta innegable de cualquier directivo, lo cual está asociado a la efectividad administrativa, donde si bien no existe una fórmula mágica para alcanzarla, sí se encuentran elementos que pueden ser garantes de un mayor acercamiento a este propósito, siendo importante integrar de forma sólida las tres dimensiones del éxito: «La impersonal lo concerniente al trabajo, la interpersonal lo que atañe a las relaciones, y la personal, la que involucra al "yo» (Kofman, 2012, p. 13). En estas dimensiones se expresan las habilidades gerenciales, donde la coherencia constituye el hilo que enlaza el desarrollo integral del ser humano, para que los diferentes 
elementos que componen la estructura interna puedan desplegarse generando vínculos relacionales positivos a todo nivel.

Reviste, por lo tanto, singular importancia las habilidades gerenciales, las cuales según se define: «Están conformadas por el conjunto de conocimientos, habilidades, destrezas, comportamientos y aptitudes que necesita una persona para ser eficiente en una amplia gama de labores gerenciales y en diversas organizaciones» (Longenecker et al., 2009, citado en Leyva et al., 2017 p. 10). Desde este aporte, es perceptible que las habilidades están enfocadas en dotar a las personas de una mayor capacidad para llevar a cabo diversas tareas y trabajos, desde un rendimiento óptimo, con resultados significativos para el logro de los objetivos. Estas tienen un comportamiento que se ajusta a cada individuo, puesto que no todos las tienen en igual presencia y desarrollo.

«En la última década, más o menos, se ha producido una abundancia de evidencia de que la administración habilidosa es el determinante fundamental para el éxito organizacional. Si las organizaciones quieren tener éxito, deben tener directivos hábiles y competentes» (Whetten \& Cameron, 2005, p. 4). De este modo, dentro de la gerencia integral, el rol de líder inicia gerenciando la propia vida y luego, cuando se alcanza una posición estratégica dentro de una organización, surge con total determinación el llamado a ser modelo, referente, a inspirar y generar valor para el buen desempeño colectivo. "Los empleados conscientes necesitan gerentes conscientes si deben aplicar de lleno su energía al logro de las metas organizacionales» (Kofman, 2012, p. 10).

Son numerosos los intentos que se han adelantado por delimitar el conjunto de habilidades que debe poseer un ejecutivo conducente al cumplimiento exitoso de las funciones a su cargo; no obstante, aunque hay un marco de referencia, es fundamental comprender que estas se encuentran determinadas por la forma como las personas logran empoderarse y gestionarlas en el contexto de sus relaciones.

Así, como menciona Leal (2020), la adecuada gestión de competencias y habilidades «hacen que el entorno de trabajo sea más flexible y más fácil de administrar a través de comportamientos sutiles y comunicación, incluyen atributos como adaptabilidad y flexibilidad, y también incluye pensamiento creativo, resolución de conflictos, gestión del tiempo y automotivación» (p. 3). Sin duda, son herramientas de valor que potencian los entornos relacionales de las personas, dotándolas de mayor capacidad para responder a diferentes demandas en sus contextos de desarrollo y, en lo particular de la labor gerencial, para gestionar exitosamente una empresa.

El gerente debe consolidar tres habilidades gerenciales básicas que son: habilidades técnicas, humanas y conceptuales. Las habilidades técnicas, se refiere a los conocimientos y las experiencias en determinados procesos. Las habilidades humanas, se enfocan en la manera en la que el gerente interactúa efectivamente con la gente. Las habilidades conceptuales se tratan de la formulación de ideas y, la resolución de problemas en forma creativa. (Aguirre, 2007, citado en Hernández et al., 2017 p. 140).

Las anteriores agrupaciones, bien pueden dar una noción de cómo se integran las habilidades para generar un desempeño competente y coherente, donde es necesario aplicar el conocimiento en diferentes circunstancias, enfrentar retos diversos y realizar una lectura 
consciente de la realidad para encauzar de la mejor forma, los recursos con los que se cuenta para ser resolutivos ante cada evento de la vida. De este modo, dentro de las habilidades gerenciales más destacadas y requeridas en la administración moderna, se encuentran las habilidades blandas, aquellas asociadas a la personalidad, las emociones y naturaleza del individuo, entre las que se destacan: liderazgo, escucha activa, empatía, creatividad, adaptación, trabajo en equipo, comunicación, negociación, toma de decisiones, entre otras.

Desde la perspectiva de Ramírez (2018): «En la actualidad, las habilidades directivas se han transformado en factores diferenciadores de eficacia y ejecución en cualquier sector empresarial y puesto directivo, por lo que es necesario un trabajo personal para llegar a desarrollarlas» (p. 24). Es incuestionable la relación proporcional que existe entre el desarrollo personal y el desarrollo organizacional para destacarse en un mundo altamente competitivo, es verdaderamente significativo saber conectarse con los demás.

En este orden de ideas, se puede hablar de un proceso de aprendizaje permanente, continuo, que inicia desde el empoderamiento individual y se extiende hacia todos los niveles relacionales de la persona.

El valor y desarrollo de la habilidad directiva exige conocer qué hacen los directivos y qué deben hacer, qué precisan para hacerlo y, sobre todo, los resultados que se espera de ellos. Para esto necesitan habilidades y destrezas interpersonales para poder motivar, liderar, guiar, influir y persuadir el trabajo en equipo (Ramírez, 2018, p. 25).
En esencia, se reafirma el hecho de lograr un desarrollo coherente, basado en la comunicación armoniosa entre el ser, el saber y el hacer, a partir de los cuales se establece la gestión consciente del tejido de relaciones. Esto innegablemente traza un hilo conector con el éxito directivo, al definir un modelo de desarrollo interconectado, donde cada persona aporta en función de lo que construye con otros. Como lo plantean García et al. (2017): «En otras palabras, las habilidades gerenciales son los bloques de construcción sobre los que descansa la administración efectiva, donde los directivos traducen su propio estilo y lo llevan a la práctica» (p. 4). De allí, que no sean estáticas, que deban estar en permanente evolución, acompañando la realización de las metas y propósitos, las proyecciones a todo nivel, más aún, reconociendo que las dinámicas complejas y exigentes de la sociedad implican dinamismo y adaptación.

Así las cosas, es importante acudir a herramientas de apoyo para el despliegue de las habilidades gerenciales. De tal manera que, «resulta acertado disponer de formación y experiencia en la metodología del coaching y otras habilidades técnicas, humanas y conceptuales necesarias, para el ejercicio de las funciones del gerente» (Cardozo, 2016, p. 127), las cuales se configuran como herramientas de valor para alcanzar de manera estratégica los objetivos organizacionales, acompañar mejor el desempeño de los miembros de su equipo y desarrollar un liderazgo efectivo. 


\section{Toma de decisiones}

\section{en las organizaciones}

Cuando se habla de toma de decisiones, se aborda un proceso que está presente en el diario vivir y se constituye en una característica común dentro de la gestión gerencial. Algunas de estas decisiones pueden cambiar el curso de la organización y definir escenarios de actuación complejos, al punto de impactar positiva o negativamente el tejido de relaciones, procesos y actividades de injerencia empresarial. Como lo expresa Amaya (2010): «Un gerente debe tomar muchas decisiones todos los días. Algunas de ellas son decisiones de rutina mientras que otras tienen una repercusión drástica en las operaciones de la empresa donde trabaja» (p. 3).

Se establece de este modo una importante consideración al proceso de toma de decisiones dentro del entorno estratégico de cada organización, pues, como bien se señala, muchas de estas pueden darse casi que, de forma espontánea, porque corresponden la mecanicidad de lo cotidiano; no obstante, otras representan verdaderos retos al momento de avanzar en la conducción de la empresa hacia niveles superiores de su trayectoria. Resulta sencillo, en este orden, reconocer los aciertos, pero cuando las cosas no salen a favor de lo esperado, siempre se busca encontrar el foco del error, así se destaca, como lo sustenta Arocha y Niega (2020), que «la mayoría de los fracasos corporativos de hoy en día surgen a partir de malas decisiones gerenciales; por una incapacidad de ver el mundo de otro modo (p. 6).

Desde este punto de vista, Canelones y Fuentes sustentan que:
El destino de las organizaciones se basa en la toma de decisiones, y cualquier actividad gerencial no puede perder la perspectiva y tampoco ignorar tales decisiones cuya tendencia siempre será la búsqueda de soluciones simples o definitivas, que pueden ser exitosas o fracasadas. (Canelones y Fuentes, 2015, p. 56).

Esto reafirma a la toma de decisiones como uno de los procesos inherentes a la gerencia, que implica determinar un marco de actuación coherente con la misión, visión y objetivos trazados por la organización, los cuales son un punto de referencia a la hora de evaluar las diferentes alternativas con las que se cuenta.

En esa misma dirección, Amaya (2010) expresa: "Con frecuencia, las decisiones de rutina se toman rápidamente, quizás inconscientemente, sin necesidad de elaborar un proceso detallado de consideración. Sin embargo, cuando las decisiones son complejas, críticas o importantes, es necesario tomarse el tiempo para decidir sistemáticamente». (Amaya, 2010 , p. 3). Así las cosas, las decisiones también tienen jerarquía y niveles, algunas corresponden a lo primario, lo cotidiano, la rutina operativa y administrativa, mientras que otras se destacan por el alto impacto y trascendencia que pueden llegar a tener en el rumbo de la organización.

En este contexto, la información constituye una herramienta garante frente a un menor riesgo e incertidumbre, pues no solo las buenas señales o la intuición deben marcar el camino de elección, es indispensable contar con elementos de valor que proporcionen enfoque hacia los mejores ambientes para la decisión. Desde esta perspectiva, Rodríguez (2015) expone que: 
La información, incide considerablemente en la toma de decisiones. Si bien los individuos desarrollan el proceso, la información es la que permite que se pueda percibir adecuadamente lo que acontece en una organización y su ambiente cuando se va a tomar determinada decisión. (Rodríguez, 2015, p. 154)

Desde esta óptica, la información amplifica el entorno perceptivo de una situación a intervenir, haciendo posible pasar de lo subjetivo y situarse en un nivel de mayor control, al disponer de información que se aporte en forma real, actualizada y procesada con precisión. Es así como lo plantea Rodríguez (2015):

La Gestión de Información y la Gestión del Conocimiento contribuyen a la toma de decisiones estratégicas pues a partir de la información y el conocimiento se pueden generar y determinar mejores alternativas de decisión y la mejor solución ante una situación-problema. (p. 162)

Por tanto, una decisión acertada parte de un proceso de fundamentación, donde el rumbo que se define implica asumir consecuencias, poniendo de manifiesto la importancia de desarrollar una amplia capacidad para integrar diferentes elementos y comprender de una forma holística lo que dinamiza una situación.

En el proceso de toma de decisiones entran en juego dos fuentes de información: 1) una fuente de información interna al individuo, que tiene que ver con sus preferencias, creencias u objetivos; y 2) una fuente de información externa, que tiene que ver con el contexto en el cual se circunscribe la decisión y define las alternativas susceptibles de elección. (Muñetón et al., 2017, p. 1)
Desde este enfoque, se entiende el aporte del coaching en estos niveles de gestión de la información. Desde lo interno, acompañando el desarrollo de capacidades para optimizar los recursos emocionales y cognitivos de la persona, y a nivel externo, desde el rol de observador, con mayor control sobre los factores que moldean la información disponible, para hacer un uso más objetivo, imparcial y transparente de estos.

Una empresa está formada por personas con diferentes historias, diferentes culturas, diferentes inclinaciones, diferentes aspiraciones, diferentes trabajos, [...] Y todas esas personas, de diferentes edades y culturas, con diferentes empleos, tienen que llevar a cabo el milagro de trabajar juntos, de modo que los resultados de la empresa sean los adecuados. (Agnelli, G., citado en Argandoña, 2011, p. 24)

De este modo, la toma de decisiones requiere estar acompañada, además de los elementos propios de la administración y operación del negocio, por un fuerte sentido de equipo, por el reconocimiento de unos valores y principios alrededor de los cuales se espera que todas las personas se integren y de esta manera lograr una visión compartida frente a lo que se pretende alcanzar. Por esto resulta razonable que la capacidad de tomar decisiones de un gerente, de manera individual, se vea sumamente restringida por sus limitaciones cognoscitivas, por la disponibilidad de tiempo y por el acceso a la información (Huber, 1980, citado en Rodríguez, 2015, p. 154). En este orden, un factor garante en el camino que guía la toma de decisiones se encuentra en la gestión de los equipos, donde confluye una importante información desde los roles específicos que adoptan en la dinámica organizacional, así es 
posible articular una visión más amplia para comprender los diferentes fenómenos y asumir con rigurosidad, un acercamiento objetivo a su estudio.

Lo anterior, plantea una responsabilidad compartida, transparente en la que cada persona se involucre con los resultados, disponiendo su potencial y talento al servicio de lo que ha sido asumido por el colectivo, para lograr niveles superiores de desarrollo. Desde allí, se reconoce de manera prevalente el valor que tiene trabajar fortaleciendo vínculos saludables, que consoliden una gestión desde el acompañamiento, donde todos puedan corresponder de manera asertiva y aportar, en su nivel de competencia, a la construcción de una organización interdependiente.

Bajo este precepto:

El proceso de decisión no solo se lleva a cabo a nivel estratégico o de la más alta dirección de las organizaciones, sino que constituye un proceso que se desarrolla en toda organización y en todos sus niveles: operativo, táctico, gerencial y estratégico. (Wiig, 2003 citado en Rodríguez, 2015, p. 152)

Por tanto, depende de cada uno el grado de consciencia que logra desarrollar para una gestión exitosa al momento de evaluar los caminos posibles con relación a la realidad que se interviene, donde toma validez que, «tanto gerentes como empleados deberían ser alentados a pensar creativa y críticamente cualquier aspecto de la organización. Se les debería alentar a cuestionarlo todo hasta que consigan mejores formas de trabajar o crear valor» (Arocha y Noriega, 2020, p. 7).
En efecto, involucrar a todas las personas de la organización en este proceso requiere un aprendizaje colectivo, con el que se pueda estar por encima de posiciones egoístas y desarticuladas de una visión compartida, por el compromiso con un proyecto organizacional, donde cada persona logre identificar el rol protagónico que puede desempeñar al conectarse con el querer y deber ser como colectivo de trabajo.

Muñetón et al., (2017) aportan: «La toma de decisión depende no solo de la acumulación de información ganada a través de la experiencia, sino también del uso que se hace y la comprensión que se tiene de la misma» (p. 6). Por esto se hace importante revestir este proceso de talento y habilidad, porque no es un tema que se pueda definir a la ligera, más aún cuando por mínima que resulte la acción, va a tener un efecto, el cual debería estar enfocado en generar el mayor equilibrio y bienestar posible, sin desconocer que hay un entorno estratégico presente. De allí, la importancia de establecer un aprendizaje continuo, en procura de lograr una mejor toma de decisiones y que sean acertadas, incluyendo la integridad, la confianza y la capacidad para demostrar que el coaching es esencial en el empoderamiento organizacional (Barrientos et al., 2019, p. 234).

Con lo expuesto, se define una importante relación entre el coaching y la toma de decisiones, destacando que un adecuado ejercicio de elección parte de la confianza y la capacidad de movilizar esquemas internos, donde las emociones se conecten con la razón, a partir de un progresivo entrenamiento personal, que bien puede y debe escalarse hacia la gestión colectiva. Desde este enfoque, lo que se busca a través del coaching es «apoyar a la gente para que estas aprendan de su propia reflexión 
y capacidades de conseguir soluciones a sus problemas y retos» (Díaz, 2016, p. 110). Esto sin duda constituye un pilar de avance en la dinámica de las organizaciones, puesto que al empoderar a las personas y acompañarlas en el desarrollo consciente de sus capacidades, se traduce en un lenguaje de conexión y pertenencia dentro de los equipos colaborativos. Este tipo de sinergia bien puede impactar de manera positiva la búsqueda integrada de soluciones, donde todos se sienten parte funcional y vital del entramado organizacional.

\section{Conclusiones}

El coaching aporta de manera significativa en la toma de decisiones gerenciales. En sí mismo es una metodología que fortalece el entorno estratégico de la organización, al punto de conducirla, mediante la transformación de sus directivos y colaboradores, hacia niveles crecientes de desarrollo; así, cada integrante alcanza un mayor equilibrio entre lo racional y lo intuitivo, una mayor observancia de los pensamientos y de las emociones. Consecuentemente, al aumentar el autodominio y autorreconocimiento, se fortalece la capacidad de accionar ante cualquier situación, evaluar la información al alcance, considerar diferentes puntos de vista, generar más de una alternativa para la solución, superar juicios y limitaciones personales, entre muchos otros, que destacan el valor de un proceso de aprendizaje que coadyuva la valoración de las capacidades individuales y colectivas, para hacer más efectivo el logro de los resultados.

La gerencia es una función vital y estratégica en toda organización, dentro de la cual está inmerso el reto de conectar a las personas y de actuar habilidosamente en la transformación positiva de los entornos. En este propósito, el coaching se configura como una herramienta de invaluable apoyo para los gerentes y líderes, mediante la conducción de procesos reflexivos y creativos, que integran a los equipos colaborativos en una dinámica de mejora continua, en tareas tan importantes como la toma decisiones, con criterio, control de riesgos y enfoque hacia la competitividad empresarial.

El coaching organizacional, además del acompañamiento y entrenamiento a la alta gerencia, se incrementa en su nivel de alcance, cuando se logra llevar el proceso de intervención a diferentes niveles de la organización. No obstante, una de las metas es dotar a los líderes de herramientas de gestión desde la consciencia, para que alcancen su desarrollo como líderes coach y emprendan procesos desde el acompañamiento efectivo con sus equipos.

La tendencia del coaching se reafirma en los profundos cambios de la sociedad actual, donde las personas perciben el llamado a descubrir sentidos de vida, potenciar experiencias memorables y lograr una mayor conexión en los entornos de interacción cotidianos. En este contexto, lo organizacional demanda resignificarse desde lo humano, empoderar a los equipos de trabajo y fortalecer liderazgos transformacionales.

Las habilidades gerenciales son imprescindibles en el mundo laboral, estas posibilitan interactuar de manera efectiva y construir un tejido de relaciones sinérgicas, elementos diferenciadores que dan soporte a la forma de dirigir, liderar y orientar los equipos de trabajo. Su desarrollo está ligado al conocimiento y manejo de las emociones, lo cual requiere profundizar en el propio "yo», acercarse al mundo interior, aprender de este y en muchas oportunidades derrumbar estructuras, para reinventarse. 
Es posible tener un mayor dominio a la hora de decidir, más cuando se logra un proceso expansivo de autorreconocimiento y conexión del potencial individual, proceso que, conducido desde el coaching, acompaña el camino de autoaprendizaje para alcanzar mayores niveles de consciencia, haciendo posible disminuir la incertidumbre al momento de su ejecución. Esto se fundamenta en mayor equilibrio entre la razón y la emoción, lo cual los dota a los gerentes, estrategas y equipos colaborativos, de una mayor competencia para manejar de forma asertiva la información disponible, evaluar las alternativas y generar confianza para la elección final.

\section{Referencias}

- Amaya, A, J. (2010). Toma de decisiones gerenciales: Métodos cuantitativos para la administración. Segunda edición, Bogotá D.C.: Ecoe Ediciones.

- Aponte Polanco, T. P. (2017). Megatendencias administrativas como alternativas estratégicas de desarrollo organizacional. Universidad Militar Nueva Granada, 1-24. https://repository.unimilitar. edu.co/handle/10654/17036

- Argandoña, A., (2011). La ética y la toma de decisiones en la empresa. Universia Business Review, (30), 22-31. https://www.redalyc.org/ articulo.oa?id=43318798002

- Arocha, C., \& Noriega, V., (2020). Mala praxis gerencial. Revista de Informaciónn Científica para la Dirección en Salud. INFODIR, 0(32). http://revinfodir.sld.cu/index.php/infodir/article/ view/849

- Arroyo, T. R., (2013). Habilidades gerenciales: desarrollo de destrezas, competencias y actitud. Bogotá, D.C: Ecoe Ediciones.
- Baquero Barato, J. A., \& Rodríguez-Moneo, M., (2016). La relación entre el proceso de autorregulación y el proceso de coaching. Universitas Psychologica, 15(1), 141-152. https:// doi.org/10.11144/Javeriana.upsy15-1.rpap

- Barrientos-Monsalve, E. J., Hurtado-Hernández, L. J., Lesmes-Silva, A. K., \& Duarte-Rey, D. M. (2019). ¿Coaching en las empresas? La gerencia del coaching en las organizaciones contemporáneas. Mundo FESC, 10(S1), 223-236. https://www.fesc.edu.co/Revistas/OJS/index. php/mundofesc/article/view/428/504

- Canelones, O. J. \& Fuentes, R., (2015). Formación gerencial, toma de decisiones un abordaje desde el punto de vista holístico. Revista Negotium, 11(31), 48-73. https://www.redalyc.org/ pdf/782/78241171004.pdf

- Díaz, C. G., (2016). El Gerente Coaching en la Gerencia. Revista Scientific, 1(2), 110-130. http://www.indteca.com/ojs/index.php/ Revista_Scientific/article/view/28/25

- Casique, F, L., (2018). Impacto del coaching organizacional en el desarrollo de competencias laborales del talento humano de empresas Manizaleñas, una mirada desde sus directivos (tesis de maestría en administración). Universidad Nacional de Colombia, Manizales. http://bdigital. unal.edu.co/69811/7/1090398867.2018.pdf

- Castillo, C. A., Medina Durán, V. K. (2016). Innovación en procesos de gestión: Una mirada al coaching en las organizaciones. Revista Apuntes de Administración, 1(1), 68-75. https:// revistas.ufps.edu.co/index.php/apadmin/article/ view/996/943

- García, F. J., Boom, E. A., \& Molina, S. J. (2017). Habilidades del gerente en organizaciones del sector palmicultor en el departamento del Cesar-Colombia. Revista Científica «Visión de Futuro», 21(2), 1-21. https://www.redalyc.org/ pdf/3579/357955446001.pdf 
- Chew, G., Wei, C., Yen, Y., Ming, W., \& Sabbir M., (2020). Executive coaching effectiveness: towards sustainable business excellence. Total Quality Management \& Business Excellence, 1-19. https://doi.org/10.1080/14783363.2020.17 24507

- González, M., De Diego, A., \& González, L. J. (2018). Mindfulness y coaching: promoviendo el desarrollo de la presencia y la conciencia plena. MLS Psychology Research, 1(1), 79-94. https://www.mlsjournals.com/ Psychology-Research-Journal/article/ view/114/281

- Hernández, Valbuena, M. E., (2016). El coaching como proceso de formación. Neumann Business Review, 2(1), 59-73. https://www.journaltop.com/ index.php/NBR/article/view/Art5

- Hernández Palma, H., Muñoz Rojas, D., \& Barrios Parejo, I. (2017). Estilos gerenciales y su influencia en la generación de valor de las instituciones prestadoras de salud de la región Caribe. Revista Económicas CUC, 38(1), 133146. https://revistascientificas.cuc.edu.co/ economicascuc/article/view/1574/pdf_125

- Kofman, F. (2012). La Empresa consciente: cómo construir valor a través de valores. Ciudad Autónoma de Buenos Aires: Editorial Aguilar.

- Leal, M. I., Mejías, P. L., \& Guédez, T. M. (2017). Coaching ontológico como enfoque gerencial para la transformación y desarrollo organizacional. Dissertare, Revista de Investigación en Ciencias Sociales, 129-138. https://150.186.96.55/index.php/dissertare/ article/view/1116/459

- Leal, M. S., (2020). Las competencias blandas en los gerentes de proyecto de las organizaciones. Res Non Verba Revista Científica, 10(1), 1-24. http://34.216.34.33/index.php/rnv/article/ view/286
- Leyva, A. B., Espejel, B. J. E., \& Cavazos, A. J., (2017). Habilidades gerenciales como estrategia de competitividad empresarial en las pequeñas y medianas empresas (Pymes). Revista Perspectiva Empresarial, 4(1), 7-22. https://revistas.ceipa.edu.co/index.php/ perspectiva-empresarial/article/view/119/54

- Muñetón, S. G., Ruiz, M. A. F., Loaiza, Q. O. L., (2017). Toma de decisiones: Explicaciones desde la ciencia aplicada del comportamiento. Revista Espacios, 38(13), 1- 12. https://revistaespacios. com/a17v38n13/a17v38n13p10.pdf

- Muñoz Maya, C. M., \& Díaz Villamizar, O. L., (2014). El Coaching y la transformación organizacional: una oportunidad para las Empresas y los Coaches. Suma Negocios, 5(11), 62-69. https://doi.org/10.1016/ S2215-910X(14)70020-5

- Musicco, G., (2015). Coaching: conciliación de vida laboral - personal (WLB). Revista del Centro de Investigación de la Universidad La Salle, 11(44), 121-142. http://52.226.65.210/index.php/ recein/article/view/684/1065

- Navia Mayorga, F. A., Mayorga Arias, D., Campi Mayorga, I. I., De Lucas Coloma. L. A., (2019). Liderazgo: una habilidad gerencial fundamental en el éxito de una empresa en el siglo XXI.

Revista Científica Mundo de la Investigación y el Conocimiento, 3(3), 1061-1084. https://dialnet. unirioja.es/servlet/articulo?codigo=7116501

- Ramírez, J. (2018). Las habilidades directivas una condición para una ejecución eficaz. Revista Investigación y Negocios, 11(17), 2329. http://www.scielo.org.bo/pdf/riyn/v11n17/ v11n17_a04.pdf

- Recuenco, C. A. D., (2018). Coaching ejecutivo: técnica gerencial necesaria en la era del conocimiento. Revista SCIÉNDO 21(1): 79-88. http://revistas.unitru.edu.pe/index.php/SCIENDO/ article/view/1727/1698 
- Rodríguez, C. Y., (2015). Gestión de Información y del Conocimiento para la toma de decisiones organizacionales. Bibliotecas anales de investigación, (11), 150-163. https://dialnet. unirioja.es/servlet/articulo?codigo $=5704545$

- Sánchez, M. B., \& Boronat, M. J., (2014). Coaching educativo: modelo para el desarrollo de competencias intra e interpersonales. Educación XX1, 17(1), 221-242. https://www.redalyc.org/ pdf/706/70629509010.pdf
- Suárez, M. M., (2020). Aplicar técnicas de coaching en la empresa: ¿Es posible y apareja resultados positivos? Revista Científica Internacional, 7(1), 237-250. http://www.utic.edu. py/revista.ojs/index.php/revista/article/view/105

- Whetten, D. A., \& Cameron, K. (2005). Desarrollo de Habilidades Directivas. (6. ${ }^{a}$ ed.). Pearson Educación. 